\title{
Structural and dynamical properties of liquid Si. An orbital-free molecular dynamics study
}

\author{
A. Delisle ${ }^{1}$, D.J. González ${ }^{2}$ and M.J. Stott ${ }^{1}$ \\ 1 Department of Physics, Queen's University, Kingston, Ontario, CANADA and \\ 2 Departamento de Física Teórica, Universidad de Valladolid, Valladolid, SPAIN
}

(Dated: November 6, 2018)

\begin{abstract}
Several static and dynamic properties of liquid silicon near melting have been determined from an orbital free ab-initio molecular dynamics simulation. The calculated static structure is in good agreement with the available X-ray and neutron diffraction data. The dynamical structure shows collective density excitations with an associated dispersion relation which closely follows recent experimental data. It is found that liquid silicon can not sustain the propagation of shear waves which can be related to the power spectrum of the velocity autocorrelation function. Accurate estimates have also been obtained for several transport coefficients. The overall picture is that the dynamic properties have many characteristics of the simple liquid metals although some conspicuous differences have been found.

PACS numbers: 61.20.Ja, 61.20.Lc, 61.25.Mv, 71.15.Pd
\end{abstract}

\section{INTRODUCTION.}

Molecular dynamics (MD) methods are a useful technique to study the properties of liquid systems, and the last two decades have witnessed a large increase in the application of ab-initio molecular dynamics methods (AIMD) based on the density functional theory ${ }^{\underline{1}}$ (DFT) for the treatment of the electron system. This theory allows calculation of the ground state electronic energy of a collection of atoms, for given nuclear positions, and also yields the forces on the nuclei via the HellmannFeynman theorem. It allows MD simulations in which the nuclear positions evolve according to classical mechanics with the electronic subsystem following adiabatically. Most AIMD methods are based on the Kohn-Sham (KS) orbital representation of the DFT (KS-AIMD methods) which, at present, poses heavy computational demands which limit the size of the systems that can be studied as well as the simulation times. However, some of these constraints are eased in the so-called orbital-free $a b$-initio molecular dynamics (OF-AIMD) method, which by disposing of the electronic orbitals of the KS formulation provides a simulation method where the number of variables describing the electronic state is greatly reduced, enabling the study of larger samples (several hundreds of particles) and for longer simulation times (tens of ps).

This paper reports the results of an ab-initio molecular dynamics simulation on the static and dynamic properties of liquid Silicon (l-Si) at thermodynamic conditions near its triple point. $\mathrm{Si}$ is an interesting material with many peculiar properties which, along with its technological importance, has stimulated intensive theoretical ${ }^{2.3 .4 .5 .6 .7 .8 .9}$ and experimental $l^{10,11,12.13 .14 .15}$ work. Its high-density forms include the crystalline, amorphous and liquid phases with the former two being covalently bonded and semiconducting and the latter one metallic. At melting Si undergoes a semiconductormetal transition along with a density increase of $\approx$ $10 \%$ and significant changes in the local atomic struc- ture. This evolves from an open one, with a tetrahedral fourfold coordination, to a more compact liquid structure with a white-tin-type arrangement preserving the local tetrahedrality and with an approximate sixfold coordination 16.17

Most theoretical studies on l-Si have focused on its static structural properties and have resorted to classical MD simulations in which the liquid system was characterized by effective interatomic potentials constructed either empirically by fitting to experimental data 2.3 , or derived from some approximate theoretical model, 4.5.6. $^{\text {. More re- }}$ cently, some KS-AIMD calculations ${ }^{7.8 .9}$ have been reported, although the results dealt with electronic and static properties only because of the computational constraints inherent in this method.

Stich et a performed the first KS-AIMD calculation for l-Si near the triple point using 64 particles, a non-local Bachelet-Hamann-Schluter type pseudopotential18 and the local density approximation (LDA) for the electronic exchange and correlation energies. Subsequently, a more comprehensive study was performed ${ }^{8}$ with a larger number of atoms (350 particles) and a spin dependent generalized gradient approximation for electron exchange and correlation. A KS-AIMD calculation has also been performed by Chelikowsky et $a^{19}$ using 64 atoms and a nonlocal, Troullier-Martins type, pseudopotential $\frac{19}{19}$ These $a b$-initio studies have provided accurate descriptions of the local liquid structure and valuable insights into the the valence electron charge densities, but, by necessity, they were too short to address dynamical properties (besides the self-diffusion coefficient) and these properties will now be the main focus of the present report which, to our knowledge, is the first ab-initio study on the dynamical properties of l-Si.

The static structure factor, $S(q)$, of l-Si has been measured by both neutron scattering (NS) ${ }^{10}$ and X-ray $(\mathrm{XR})^{11.12 .13}$ diffraction. Although all experimental $S(q)$ 's show a distinctive shoulder at the high- $q$ side of the main peak, there are some discrepancies in the positions and 
heights of the first peak, its shoulder and the second peak. Experimental investigations of the dynamic structure of l-Si have been hampered by the large adiabatic sound speed ( $\approx 4000 \mathrm{~m} / \mathrm{s}$ ), which prevents the use of the inelastic thermal neutron scattering (INS) technique for investigating the collective excitations for small $q$-values. Nevertheless, the dynamical structure of 1-Si near the triple point has been investigated recently by Hosokawa et $a^{114.15}$ using high-resolution inelastic X-ray scattering (IXS) which has no such kinematic restrictions. The measurements by Hosokawa et al investigated the wavevector regions $0.02 \AA^{-1} \leq q \leq 1.3 \AA^{-1}$ [14] and $0.02 \AA^{-1} \leq q \leq$ $2.96 \AA^{-1}[15]$ and several dynamical features already observed in the liquid alkali metals were found, such as the existence of collective excitations up to $q$-values around $0.5 q_{p}$ (where $q_{p}$ is the main peak's position of $S(q)$ ), along with a positive dispersion in the adiabatic sound velocity with respect to the hydrodynamic value.

Section II contains a brief description of the orbitalfree ab-initio molecular dynamics (OF-AIMD) method, and gives some technical details particularly on the electronic kinetic energy functional and the local pseudopotential used to characterize the ion-electron interaction. In section [II we present and discuss the results of the $a b$ initio simulations for several static and dynamic properties which are compared with the available experimental data. Finally, conclusions are drawn and possible avenues for further study are suggested.

\section{THEORY.}

A liquid simple metal may be treated as a disordered array of $N$ bare ions with valence $\mathrm{Z}$, enclosed in a volume $V$, and interacting with $N_{\mathrm{e}}=N Z$ valence electrons through an electron-ion potential $v(r)$. The total potential energy of the system can be written, within the Born-Oppenheimer approximation, as the sum of the direct ion-ion coulombic interaction energy and the ground state energy of the electronic system under the external potential created by the ions, $V_{\text {ext }}\left(\vec{r},\left\{\vec{R}_{l}\right\}\right)=$ $\sum_{i=1}^{N} v\left(\left|\vec{r}-\vec{R}_{i}\right|\right)$,

$$
E\left(\left\{\vec{R}_{l}\right\}\right)=\sum_{i<j} \frac{Z^{2}}{\left|\vec{R}_{i}-\vec{R}_{j}\right|}+E_{g}\left[\rho_{g}(\vec{r}), V_{\mathrm{ext}}\left(\vec{r},\left\{\vec{R}_{l}\right\}\right)\right]
$$

where $\rho_{g}(\vec{r})$ is the ground state electronic density and $\vec{R}_{l}$ are the ion positions. According to DFT, the ground state electronic density, $\rho_{g}(\vec{r})$, can be obtained by minimizing the energy functional

$$
E[\rho(\vec{r})]=T_{s}[\rho]+E_{H}[\rho]+E_{\mathrm{xc}}[\rho]+E_{\mathrm{ext}}[\rho]
$$

where the terms represent, respectively, the electron kinetic energy, $T_{s}[\rho]$, of a non-interacting system of density $\rho(\vec{r})$, the classical electrostatic energy of the electrons (Hartree term),

$$
E_{H}[\rho]=\frac{1}{2} \iint d \vec{r} d \vec{s} \frac{\rho(\vec{r}) \rho(\vec{s})}{|\vec{r}-\vec{s}|}
$$

the exchange-correlation energy, $E_{\mathrm{xc}}[\rho]$, for which we have used the local density approximation and finally, the electron-ion interaction energy, $E_{\text {ext }}[\rho]$, where the electron-ion potential has been characterized by a first principles local pseudopotential constructed within DFT $^{20}$.

$$
E_{\mathrm{ext}}[\rho]=\int d \vec{r} \rho(\vec{r}) V_{\mathrm{ext}}(\vec{r})
$$

In the KS-AIMD method, $\frac{1}{1} T_{s}[\rho]$ is exactly evaluated by using a set of roughly $N_{\mathrm{e}}$ single particle orbitals, but at huge computational expense. In contrast, the OFAIMD approach uses an approximate but explicit density functional for $T_{s}[\rho]$ so that the system is described in terms of the single function $\rho(\vec{r})$ replacing the large set of orbitals $1,20,21$ Proposed functionals incorporate the von Weizsäcker term,

$$
T_{W}[\rho(\vec{r})]=\frac{1}{8} \int d \vec{r}|\nabla \rho(\vec{r})|^{2} / \rho(\vec{r})
$$

plus further terms chosen in order to reproduce correctly some exactly known limits. Here, we have used an average density model ${ }^{20}$, where $T_{s}=T_{W}+T_{\alpha}$,

$$
\begin{array}{r}
T_{\alpha}=\frac{3}{10} \int d \vec{r} \rho(\vec{r})^{5 / 3-2 \alpha} \tilde{k}(\vec{r})^{2} \\
\tilde{k}(\vec{r})=\left(2 k_{F}^{0}\right)^{3} \int d \vec{s} k(\vec{s}) w_{\alpha}\left(2 k_{F}^{0}|\vec{r}-\vec{s}|\right)
\end{array}
$$

$k(\vec{r})=\left(3 \pi^{2}\right)^{1 / 3} \rho(\vec{r})^{\alpha}, k_{F}^{0}$ is the Fermi wavevector for mean electron density $\rho_{e}=N_{e} / V$, and $w_{\alpha}(x)$ is a weight function chosen so that both the linear response theory and Thomas-Fermi limits are correctly recovered. Further details are given in Ref. [20].

Another key ingredient of the energy functional is the the local ionic pseudopotential, $v_{p s}(r)$, describing the ionelectron interaction. It has been constructed from firstprinciples by fitting, within the same $T_{s}[\rho]$ functional, to the displaced electronic density induced by an ion embedded in a metallic medium as obtained in a KS-DFT calculation. Further details are given in Ref. [20] and we merely note that the theoretical framework used in this study has provided an accurate description of several static and dynamic properties in the liquid $\mathrm{Li}, \mathrm{Mg}$, $\mathrm{Al}, \mathrm{Na}-\mathrm{Cs}$ and Li-Na systems ${ }^{20,22}$. 


\section{RESULTS}

OF-AIMD simulations have been performed for l-Si in a thermodynamic state characterized by the temperature $\mathrm{T}=1740 \mathrm{~K}$ and ionic number density $\rho_{i}=0.05551 \AA^{-3}$ 11]. We have considered 2000 ions in a cubic cell with periodic boundary conditions. Given the ionic positions at time $t$, the electron energy functional is minimized with respect to $\rho(\vec{r})=\psi(\vec{r})^{2}$, where $\psi(\vec{r})$ is a single effective orbital which is expanded in plane waves which are truncated at a cutoff energy, $E_{\mathrm{Cut}}=12.75$ Ryd. The energy minimization with respect to the Fourier coefficients of $\psi(\vec{r})$ is performed every ionic time step by using a quenching method which results in the ground state electronic density and energy. The forces on the ions are obtained from the electronic ground state via the HellmanFeynman theorem, and the ionic positions and velocities are updated by solving Newton's equations, with the Verlet leapfrog algorithm with a timestep of $3.5 \times 10^{-3}$ ps. In the simulations equilibration lasted $10 \mathrm{ps}$. and the calculation of properties was made averaging over 50 ps. For comparison we mention that the previous KS-AIMD simulations lasted for $1.2 \mathrm{ps}$. 7] $0.9 \mathrm{ps}$. [8] and $1.0 \mathrm{ps}$. [9], which underscores its limitations for dealing with the dynamical properties.

Several liquid static properties have been evaluated (pair distribution function, static structure factor and bond angle distribution) as well as various dynamic properties, both single-particle ones (velocity autocorrelation function, mean square displacement) and collective ones (intermediate scattering functions, dynamic structure factors, longitudinal and transverse currents). The calculation of the time correlation functions was performed by taking time origins every five time steps. Several of these functions also depend on wave vector, which for our isotropic system is a dependence on $q \equiv|\mathbf{q}|$ only.

\section{A. Static properties.}

The pair distribution function, $g(r)$, and the static structure factor $S(q)$ can both be obtained directly from the simulation. The latter is plotted in Fig. 11along with the X-ray diffraction data of Waseda et $a^{l^{11.12}}$ and the neutron data of Gabathuler and Steeb10.

The OF-AIMD $S(q)$ has a main peak at $q_{p} \approx 2.59 \AA^{-1}$ with a distinctive shoulder at $\approx 3.20 \AA^{-1}$, the second peak is at $\approx 5.65 \AA^{-1}$ and the subsequent oscillations are rather weak. As noted earlier there are some discrepancies among the measured structures in $q_{p}, S\left(q_{p}\right)$, and the positions of the shoulder and the second peak. The experimental results for $q_{p}$ range from $2.49 \AA^{-1}[13]$ to $2.78 \AA^{-1}$ [10], the position of the shoulder from 3.15 $\AA^{-1}\left[13\right.$ to $3.40 \AA^{-1}[10$, and the second peak's position from $5.53 \AA^{-1}$ [13] to $5.70 \AA^{-1}[10]$. In all cases the smaller estimate is from Takeda's XR data 13 and the larger from the NS data of Gabathuler and Steeb ${ }^{10}$. It is worth mentioning that the XR data by Waseda et $a^{111,12}$

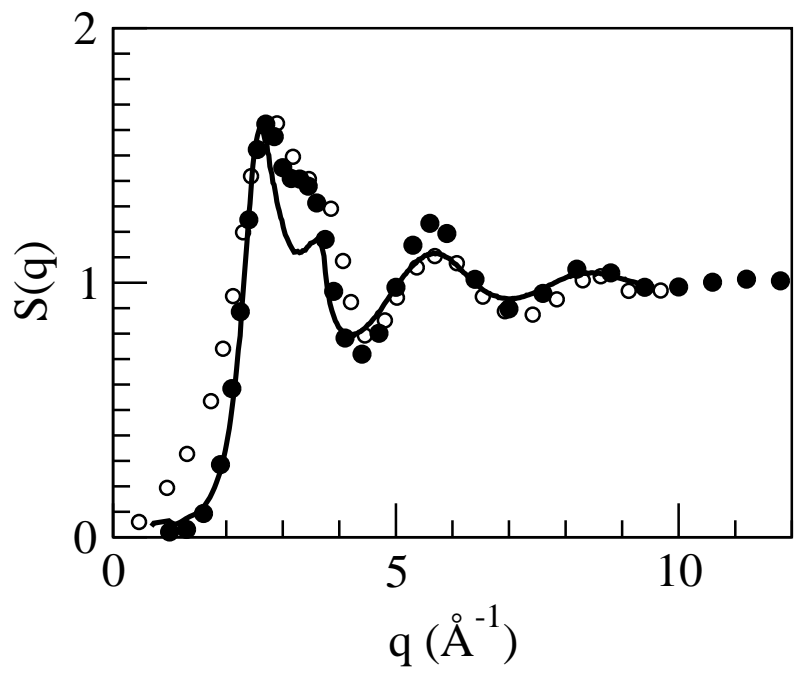

FIG. 1: Static structure factor of l-Si at 1740 K. Open circles: experimental NS data ${ }^{10}$. Full circles: experimental XR data ${ }^{11.12}$. Continuous line: OF-AIMD simulations.

gave values closer to the NS ones, namely $q_{p}=2.76 \AA^{-1}$ with the shoulder at $3.35 \AA^{-1}$ and the second peak's position at $5.66 \AA^{-1}$. Nevertheless, the values from the OF-AIMD simulation always lie within the range covered by the experimental data.

The OF-AIMD $S(q)$ accounts for the position and height of the main peak, although the amplitudes of the following peaks and troughs are somewhat underestimated. Moreover, the shoulder on the high- $q$ side of the main peak, which is the most distinctive feature of the measured $S(q)$ for l-Si, is also reproduced by the simulation although with a smaller height. A similar shortcoming is visible in the $S(q)$ from the KS-AIMD simulations of Stich et $a^{77.8}$, whereas that of Chelikowsky et $a^{79}$ gives a shoulder of similar height as the main peak.

Extrapolation of $S(q)$ to $q \rightarrow 0$ and use of the relation $S(0)=\rho_{i} k_{B} T \kappa_{T}$ gives an estimate for the isothermal compressibility $\kappa_{T}$. A least squares fit of $S(q)=s_{0}+s_{2} q^{2}$ to the calculated $S(q)$ for $q$-values up to $0.8 \AA^{-1}$ yields $S(0)=0.04 \pm 0.005$ and $\kappa_{T}=3.0 \pm 0.6\left(\right.$ in $10^{-11} \mathrm{~m}^{2}$ $\mathrm{Nw}^{-1}$ units) for $\mathrm{T}=1740 \mathrm{~K}$. This latter value stands remarkably close to the experimental result ${ }^{23}$ of $2.8 \mathrm{x}$ $10^{-11} \mathrm{~m}^{2} \mathrm{Nw}^{-1}$ and therefore provides confidence in the small- $q$ behaviour of our calculated $S(q)$.

The simulation directly gives the pair distribution function, $g(r)$, whose main peak's position, $r_{p}$, is usually identified with the average nearest neighbor distance. The OF-AIMD simulation gives $r_{p} \approx 2.43 \AA$, which is slightly smaller than the experimental data of $2.45 \AA 1112], 2.47 \AA[13]$ and $2.50 \AA[10]$. An estimate of the number of nearest neighbors is obtained by integrating the radial distribution function (RDF), $4 \pi r^{2} \rho_{i} g(r)$, up to a distance $r_{m}$ taken as the position of its first minimum 24,25 ; the present OF-AIMD study gives $r_{m} \approx$ $2.95 \AA$, yielding to a coordination number of $\approx 6.0$ atoms, 


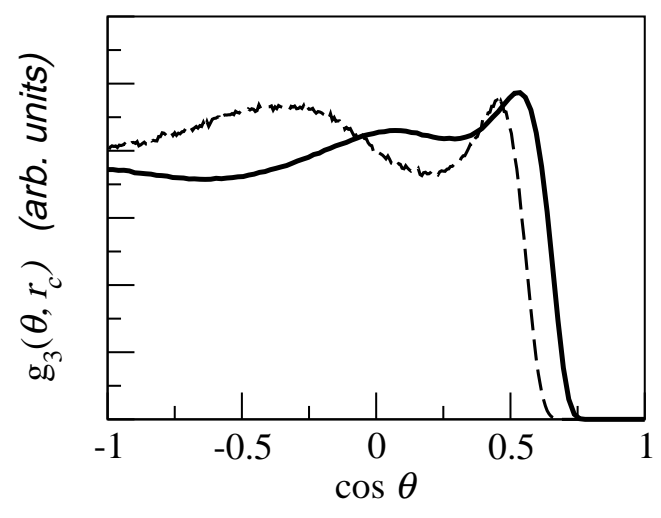

FIG. 2: Bond-angle distribution function, $g_{3}\left(\theta, r_{c}\right)$. The cutoff distance $r_{c}$ is (a) the first minimum of the RDF, $r_{c}=2.95$ $\AA$ (continuous line) and (b) the covalent cutoff $r_{c}=2.49 \AA$ (dashed line).

which reasonably compares with the experimental values at $\mathrm{T}=1735 \mathrm{~K}$ ranging from $\approx 6.4$ atoms $^{11}$ to $\approx 5.7$ atoms 13 .

Signatures of possible remnants of covalent bonding that may remain in l-Si should be contained in higher order correlation functions. A convenient quantity is the bond-angle distribution function, $g_{3}\left(\theta, r_{c}\right)$, where $\theta$ is the angle between two vectors joining a reference particle with two neighboring particles at a distance less than $r_{c}$. In liquid simple metals, this function peaks at around $\theta$ $\approx 60^{\circ}$ and $120^{\circ}$, which are close to those expected for a local icosahedral arrangement ${ }^{26}\left(\theta \approx 63.5^{\circ}\right.$ and $\left.116.5^{\circ}\right)$. The OF-AIMD results for $g_{3}\left(\theta, r_{c}\right)$, with $r_{c}$ taken as the first minimum of the RDF, are plotted in Fig. 2 where we see two maxima centered around $\theta \approx 60^{\circ}$ and $88^{\circ}$, which practically coincide with the values obtained from the KS-AIMD simulations ${ }^{7.8 .9}$, namely $\theta \approx 60^{\circ}$ and $90^{\circ}$. This double-peak feature has usually been interpreted as a signature of both tetrahedrally bonded and higher coordinated atoms contributing to the first coordination shell;

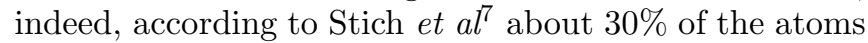
in the first coordination shell are covalently bonded. Also plotted in Fig. 2 is the $g_{3}\left(\theta, r_{c}\right)$ with $r_{c}=2.49 \AA$ which has been identified ${ }^{7.8}$ as the valence cutoff distance for covalent bonds. Now, the OF-AIMD results show a sharp maximum at $\theta \approx 65^{\circ}$ along with broader maximum centered around the tetrahedral angle $\theta \approx 109^{\circ}$. Summing up, the previous results emphasize the capability of the OF-AIMD method to account, albeit not so accurately as the KS-AIMD methods, for the orientational correlations in those liquid systems with some remnants of covalent bonding.
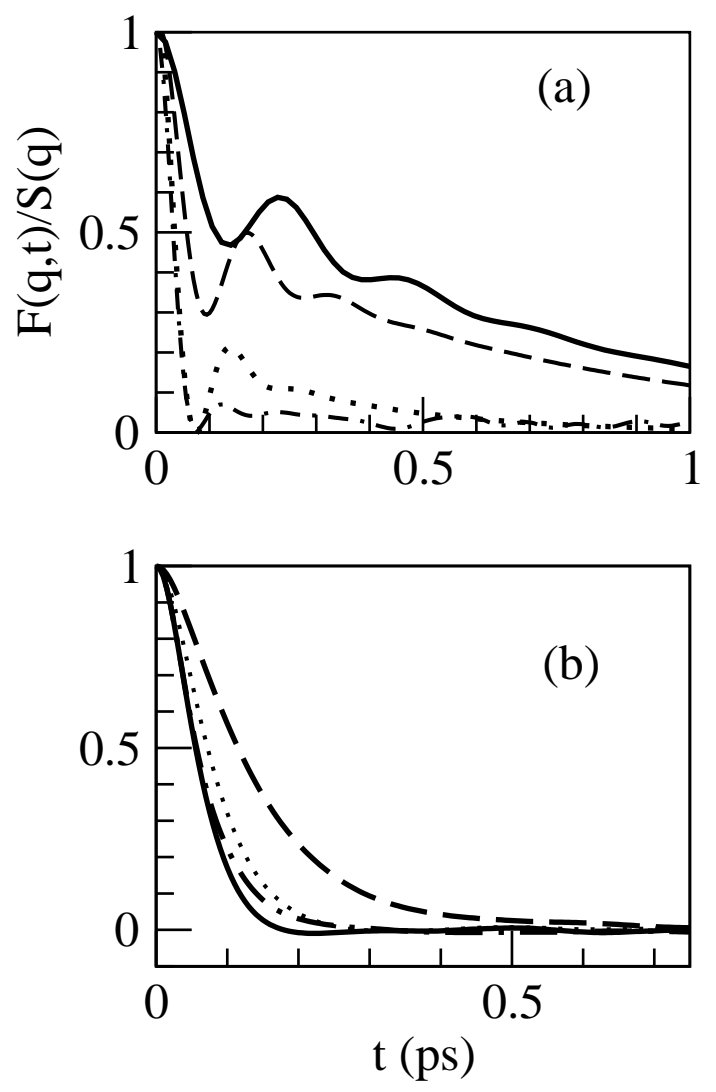

FIG. 3: Normalized intermediate scattering functions, $F(q, t)$, at several $q$-values (in $\AA^{-1}$ units), for l-Si at $\mathrm{T}=1740 \mathrm{~K}$. (a) $\mathrm{q}=0.46$ (full line), $\mathrm{q}=0.72$ (dashed line), $\mathrm{q}=1.07$ (dotted line) and $\mathrm{q}=1.46$ (dot-dashed line). (b) $\mathrm{q}=2.0$ (full line), $\mathrm{q}=2.57$ (dashed line), $\mathrm{q}=3.0$ (dotted line) and $\mathrm{q}=3.62$ (dot-dash line).

\section{B. Dynamic properties.}

\section{Collective dynamics.}

The intermediate scattering function, $F(q, t)$, defined as

$$
F(q, t)=\frac{1}{N}\left\langle\left(\sum_{j=1}^{N} e^{-i \vec{q} \vec{R}_{j}\left(t+t_{0}\right)}\right)\left(\sum_{l=1}^{N} e^{i \vec{q} \vec{R}_{l}\left(t_{0}\right)}\right)\right\rangle
$$

contains spatial and temporal information on the collective dynamics of density fluctuations. Results for $F(q, t)$ as functions of time for for several $q$-values are shown in Fig. 3] $F(q, t)$ oscillates up to $q \approx(3 / 5) q_{p}=1.55 \AA^{-1}$, with the amplitude of the oscillations being stronger for the smaller $q$-values. This behaviour, which is plotted in Fig. 3(a), is typical of simple liquid metals near melting, as found from both computer simulations ${ }^{20,27,28,29}$ and theoretical models ${ }^{30}$ However, at low $q$-values $(q \leq 0.5$ $\left.q_{p}\right)$ the $F(q, t)$ shows a strong diffusive component which plays a dominant role and imposes a slow decay. This is at variance with the results for the simple liquid metals 


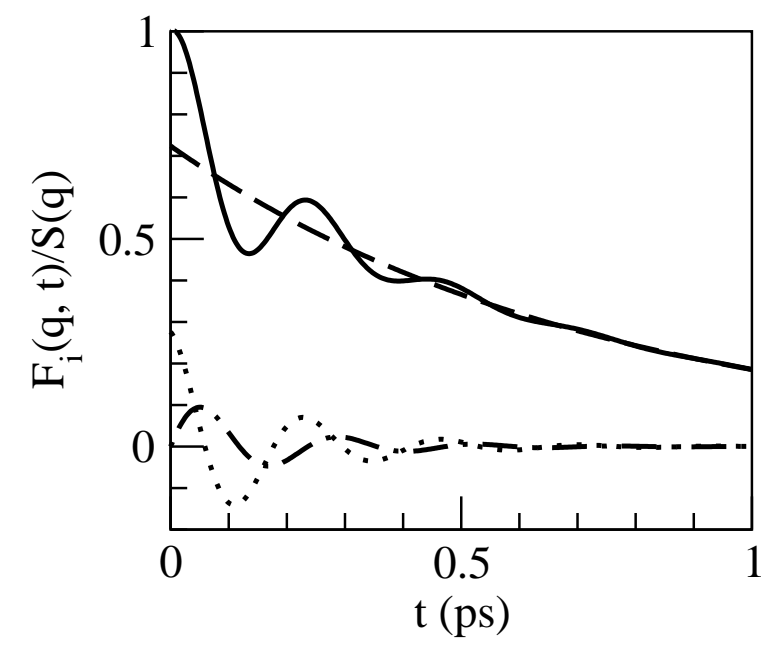

FIG. 4: Contributions to the $F(q, t)$ at $q=0.47 \AA^{-1}$ for l-Si at $\mathrm{T}=1740 \mathrm{~K}$. Diffusive component (dashed line), cosine component (dotted line) and sine component (dash-dotted line).

near melting, ${ }^{20,27,28,29,30}$ where for a comparable $q$-range the diffusive component is already very weak and the corresponding $F(q, t)$ shows marked oscillations around zero.

This different behaviour can be explained in terms of the hydrodynamic expression for the $F(q, t)$, which is exact in the $q \rightarrow 0$ limit 24.26 (hydrodynamic limit)

$$
\begin{aligned}
& F(q, t) / S(q)=\left(\frac{\gamma-1}{\gamma}\right) \exp \left(-D_{T}(q) t\right)+ \\
& \frac{1}{\gamma} \exp (-\Gamma(q) t)\left[\cos \left(c_{s} q t\right)+b q \sin \left(c_{s} q t\right)\right]
\end{aligned}
$$

and its time FT, the dynamic structure factor, $S(q, \omega)$,

$$
\begin{aligned}
& 2 \pi S(q, \omega) / S(q)=\left(\frac{\gamma-1}{\gamma}\right) \frac{2 D_{T}(q)}{\omega^{2}+\left(D_{T}(q)\right)^{2}}+ \\
& \frac{1}{\gamma}\left(\frac{\Gamma(q)+b q\left(\omega+c_{s} q\right)}{\left(\omega+c_{s} q\right)^{2}+(\Gamma(q))^{2}}+\frac{\Gamma(q)+b q\left(\omega-c_{s} q\right)}{\left(\omega-c_{s} q\right)^{2}+(\Gamma(q))^{2}}\right)
\end{aligned}
$$

where $\gamma=C_{p} / C_{v}$ is the ratio of specific heats, $c_{s}$ is the adiabatic sound velocity, $\Gamma(q)=\Gamma q^{2}$ with $\Gamma$ the sound attenuation constant and $D_{T}(q)=D_{T} q^{2}$ where $D_{T}=$ $\kappa_{T} /\left(\rho_{i} C_{p}\right)$ is the thermal diffusivity and $\kappa_{T}$ is the thermal conductivity. Finally, $\Gamma=\frac{1}{2}\left[a(\gamma-1) / \gamma+\nu_{l}\right]$ with $a=$ $\kappa_{T} /\left(\rho_{i} C_{v}\right)$ and $\nu_{l}$ is the kinematic longitudinal viscosity.

According to Eq. (9), the hydrodynamic $S(q, \omega)$ has two (inelastic) propagating peaks centered at $\omega= \pm c_{s} q$ with half-width at half-maximum (HWHM), $\Gamma(q)$, and a diffusive peak at $\omega=0$ and whose width is determined by the thermal diffusivity, $D_{T}$. For a metallic system, $D_{T}$ has electronic and ionic contributions with the former dominating; however it has been shown ${ }^{31}$ that Eqs.

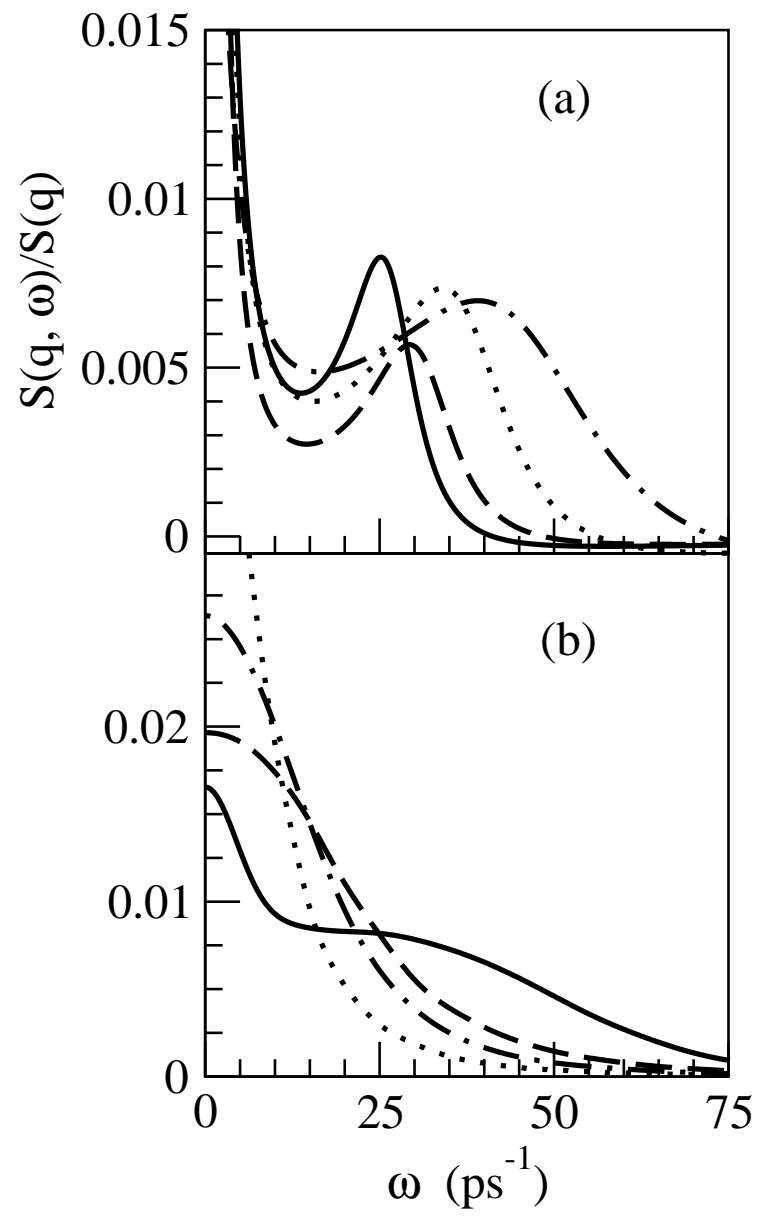

FIG. 5: Dynamic structure factor $S(k, \omega)$ at several $q$-values (in $\AA^{-1}$ units), for l-Si at $\mathrm{T}=1740 \mathrm{~K}$. (a) $\mathrm{q}=0.46$ (full line), $\mathrm{q}=0.58$ (dashed line), $\mathrm{q}=0.72$ (dotted line) and $\mathrm{q}=1.07$ (dotdashed line). (b) $q=1.46$ (full line), $q=2.00$ (dashed line), $\mathrm{q}=2.57$ (dotted line) and $\mathrm{q}=3.00$ (dot-dash line).

(8) - (9) incorporate just that part of $D_{T}$ due to the ions. Consequently, we have fitted the OF-AIMD $F(q, t)$ 's at the smallest $q$-values $\left(q \leq 0.6 \AA^{-1}\right)$, to the analytical expression of Eq. (8). A good fit was achieved in that $q$-range giving an approximate value $D_{T} \sim 1.0 \times 10^{-3}$ $\mathrm{cm}^{2} / \mathrm{sec}$., which is two orders of magnitude smaller than the experimental one ${ }^{32} D_{T}=0.228 \pm 0.004 \mathrm{~cm}^{2} / \mathrm{sec}$. for l-Si at melting, which obviously includes both ionic and electronic contributions. We note that an KS-AIMD study for liquid Ge near melting 31 gave a comparable estimate of the ionic contribution, namely $D_{T} \sim 1.3 \times 10^{-3}$ $\mathrm{cm}^{2} /$ sec., which is also two orders of magnitude smaller than its total $D_{T}$. On the other hand, estimates of the ionic contribution to $D_{T}$ in the liquid alkali metals near melting 33 range from $20.0 \times 10^{-3} \mathrm{~cm}^{2} / \mathrm{sec}$. (Li) to 3.0 $\mathrm{x} 10^{-3} \mathrm{~cm}^{2} / \mathrm{sec}$. (Cs). Consequently, as $D_{T}$ determines the diffusive behaviour of the $F(q, t)$ at small $q$ 's (see Fig. 4), the larger $D_{T}$ values of the alkali metals imply a much weaker diffusive component which is easily overcome by 


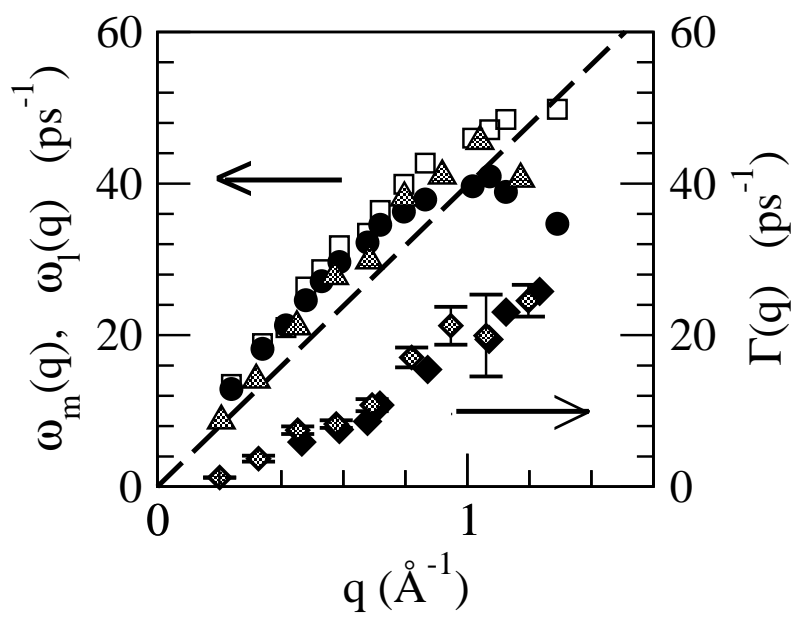

FIG. 6: Dispersion relation for l-Si at T $=1740 \mathrm{~K}$. Full circles: peak positions, $\omega_{m}(q)$, from the calculated $S(q, \omega)$. Triangles: experimental $\omega_{m}(q)$, from Hosokawa et al (Ref. 14). Open squares: peak positions, $\omega_{l}(q)$, from the maxima of the calculated longitudinal current, $J_{l}(q, \omega)$. Dashed line: Linear dispersion with the hydrodynamic sound velocity, $\mathrm{v}=3977 \mathrm{~m} / \mathrm{s}$. Grey (full) diamonds with error lines: experimental (calculated) values for the $\operatorname{HWHM}, \Gamma(q)$, of the inelastic peaks.

the oscillatory parts of the $F(q, t)$.

$S(q, \omega)$, which is the quantity directly obtained from INS or IXS experiments, has been obtained by a time FT of the $F(q, t)$ and Fig. 5 shows several $S(q, \omega)$ as functions of $\omega$ for different wavevectors up to $\approx 1.2 q_{p}$. The $S(q, \omega)$ show well defined sidepeaks, indicative of collective density excitations, up to $q \approx(3 / 5) q_{p}$ which is also a common feature of the liquid simple metals ${ }^{20.26}$.

The dispersion relation of the density fluctuations, $\omega_{m}(q)$, has been obtained from the positions of the sidepeaks of $S(q, \omega)$, and is shown in Fig. [6] along with $\omega_{l}(q)$, which is the dispersion relation obtained from the maxima of the longitudinal current correlation function, $J_{l}(q, \omega)=\omega^{2} S(q, \omega)$. Figure [6] also includes the experimental $\omega_{m}(q)$ data of Hosokawa et $a^{114}$ and a line representing the dispersion of the hydrodynamic sound whose slope gives the experimental ${ }^{34}$ adiabatic sound velocity $c_{s}=3977 \mathrm{~m} / \mathrm{s}$ at $\mathrm{T}=1753 \mathrm{~K}$. The experimental $\omega_{m}(q)$ data has a positive dispersion, i.e. an increase of $\omega_{m}(q)$ with respect to the linear hydrodynamic dispersion relation value, with a maximum located around $0.8 \AA^{-1}$ and whose magnitude amounts to $\approx 15 \%$. Although there is fair agreement between our calculated $\omega_{m}(q)$ dispersion relation and experiment, the uncertainty at the small $q$ values prevents us from unequivocally declaring positive dispersion. A similar positive dispersion has been experimentally observed in the liquid alkali metals ${ }^{35.36 .37}$, $\mathrm{Mg}^{38}, \mathrm{Al}^{35}$ and $\mathrm{Hg}^{39}$. On the other hand, we recall that at the hydrodynamic region, the slope of the dispersion relation curve gives a $q$-dependent adiabatic sound velocity, $c_{s}(q)$, which in the limit $q \rightarrow 0$ reduces to the bulk adiabatic sound velocity.
Another quantity characterizing the collective density excitations is the HWHM, $\Gamma(q)$, proportional to the inverse lifetime of the excitations. Figure [6 shows the experimental data for $\Gamma(q)$, which at low $q$ values is $\propto q^{2}$ (hydrodynamic limit) but departs from it as $q$ increases ${ }^{14.15}$. The figure also includes the $\Gamma(q)$ obtained from the simulation, which agrees fairly well with the experiment. In passing we note that to calculate $\Gamma(q)$, the $F(q, t)$ obtained from the simulation is fit to an eightparameter analytical expression that interpolates among the ideal gas, viscoelastic and hydrodynamic models 40 . This method allows the different contributions to $S(q, \omega)$ to be disentangled and gives an estimate of $\Gamma(q)$ which in the present calculations is within an error of $\approx 15 \%$.

The transverse current correlation function, $J_{t}(q, t)$, is not associated with any measurable quantity and can only be determined from computer simulations. It provides information on the shear modes and its shape evolves from a gaussian, in both $q$ and $t$, at the free particle $(q \rightarrow \infty)$ limit, to a gaussian in $q$ and exponential in $t$ at the hydrodynamic limit $(q \rightarrow 0)$, i.e.

$$
J_{t}(q \rightarrow 0, t)=\frac{1}{\beta m} e^{-q^{2} \eta|t| / m \rho_{i}},
$$

where $\eta$ is the shear viscosity coefficient, $\beta=\left(k_{B} T\right)^{-1}$ and $m$ is the atomic mass. In both the above limits $J_{t}(q, t)$ is positive, but at intermediate $q$-values there is usually a more complicated behavior with well-defined oscillations within a limited $q$-range $e^{20,26.41} . \quad J_{t}(q, t)$ as functions of time for l-Si have been obtained from the simulation and are shown in Figure $[7$ for several $q$ values up to $q_{p}$. The most striking feature is the extreme weakness of the oscillations around zero. This is in remarkable contrast to the enormous amount of MD results for different liquid systems such as hard sphere systems $\frac{41}{1}$, Lennard-Jones liquids $\frac{26.41}{}$ and simple liquid metals 20.26 .29 near melting, where the $J_{t}(q, t)$ exhibit strong oscillations and the associated spectra, $J_{t}(q, \omega)$, have an inelastic peak which appears at low $q$-values and lasts for a finite $q$-range. Again, l-Si near melting shows a different behaviour with the corresponding $J_{t}(q, \omega)$, which is also plotted in Figure 7 exhibiting a monotonic decreasing behaviour at all $q$ 's. Taking into account that the inelastic peak in the $J_{t}(q, \omega)$, is associated with propagating shear waves, the results above point to their absence in l-Si near melting.

An estimate of the shear viscosity coefficient, $\eta$, has been made from $J_{t}(q, t)$ as follows ${ }^{20.42 .43}$ The memory function representation of $J_{t}(q, t)$ :

$$
\tilde{J}_{t}(q, z)=\frac{1}{\beta m}\left[z+\frac{q^{2}}{\rho_{i} m} \tilde{\eta}(q, z)\right]^{-1},
$$

where the tilde denotes the Laplace transform, introduces a generalized shear viscosity coefficient, $\tilde{\eta}(q, z)$. The area under the normalized $J_{t}(q, t)$, gives $\beta m \tilde{J}_{t}(q, z=0)$ from 

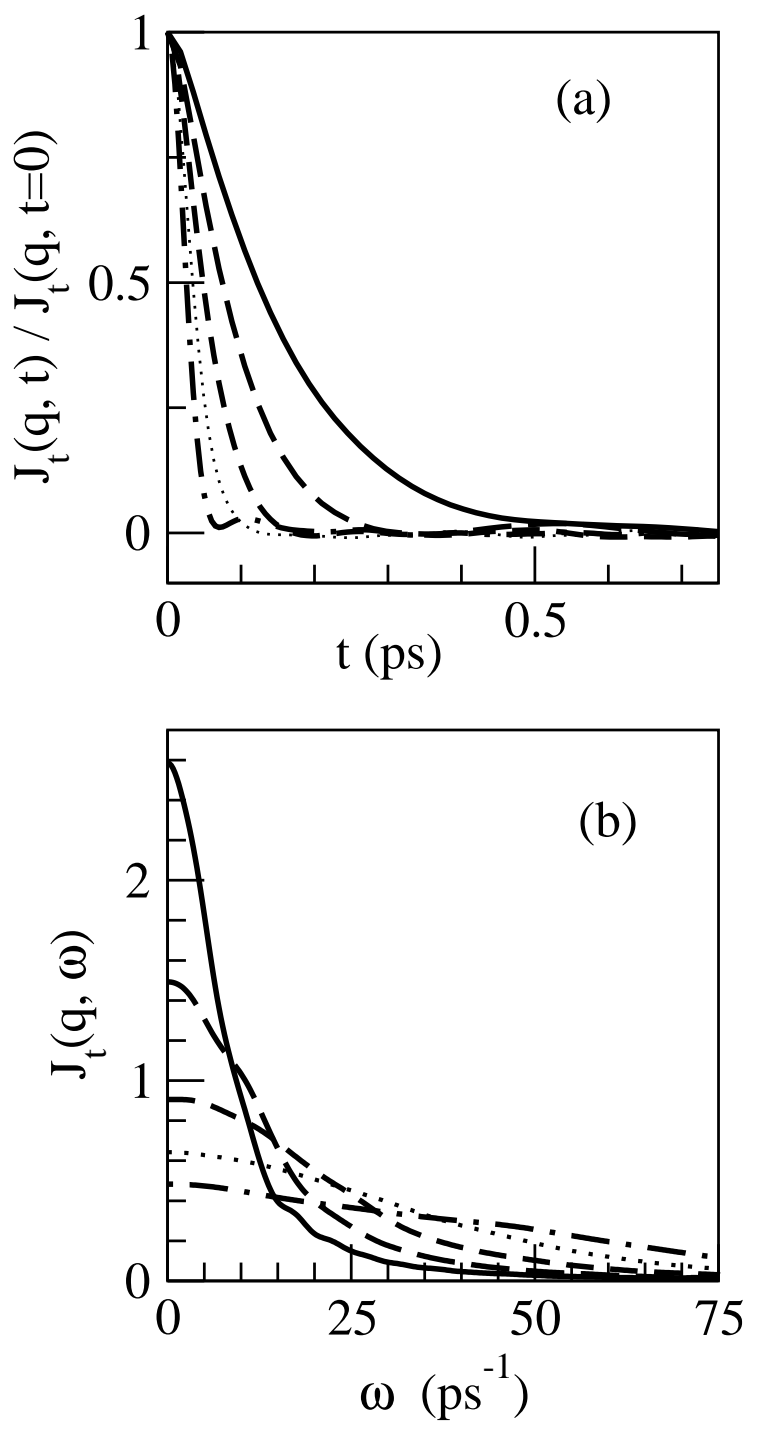

FIG. 7: (a) Transverse current correlation function $J_{t}(\mathrm{q}, \mathrm{t})$ at several $q$-values (in $\AA^{-1}$ units), for l-Si at $\mathrm{T}=1740 \mathrm{~K}$. $\mathrm{q}=0.46$ (full line), $\mathrm{q}=0.72$ (long dashed line), $\mathrm{q}=1.07$ (shortdashed line), $\mathrm{q}=1.46$ (dotted line) and $\mathrm{q}=2.57$ (dash-dotted line). (b) Same for $J_{t}(\mathrm{q}, \omega)$.

which values for $\tilde{\eta}(q, z=0)$ can be obtained and these may be extrapolated to $q=0$ to give the usual shear viscosity coefficient, $\eta$. The OF-AIMD simulations give $\eta=0.75 \pm 0.1 \mathrm{GPa} \cdot \mathrm{ps}$, which fairly compares with the available experimental results ${ }^{44} \eta_{\text {exp }}=0.58-0.78 \mathrm{GPa} \cdot \mathrm{ps}$.

\section{Single-particle dynamics.}

The most comprehensive information on the singleparticle properties is provided by the self-intermediate scattering function, $F_{s}(q, t)$, which probes the singleparticle dynamics over different length scales, ranging from the hydrodynamic to the free-particle limit. In the
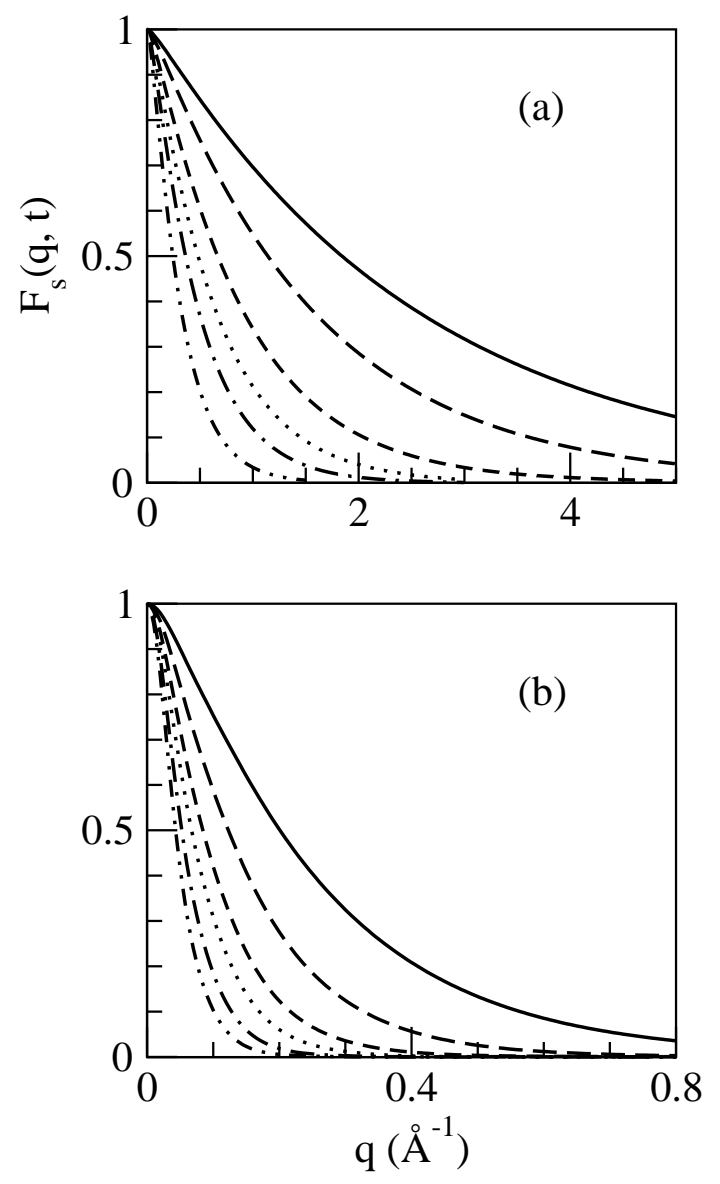

FIG. 8: Self intermediate scattering functions, $F_{s}(q, t)$, at several $q$-values (in $\AA^{-1}$ units), for l-Si at $1740 \mathrm{~K}$. (a) $\mathrm{q}=0.42$ (full line), $\mathrm{q}=0.54$ (dashed line), $\mathrm{q}=0.72$ (short dashed line), $\mathrm{q}=0.86$ (dotted line), $\mathrm{q}=1.02$ (dot-dash line) and , $\mathrm{q}=1.29$ (doble dot-dashed line). (b) $q=1.46$ (full line), $q=2.0$ (dashed line), $\mathrm{q}=2.57$ (short dashed line), $\mathrm{q}=3.0$ (dotted line), $\mathrm{q}=3.6$ (dot-dash line) and , $\mathrm{q}=4.2$ (doble dot-dashed line).

present simulations, this quantity has been obtained using

$$
F_{s}(q, t)=\frac{1}{N}\left\langle\sum_{j=1}^{N} e^{-i \vec{q} \vec{R}_{j}\left(t+t_{0}\right)} e^{i \vec{q} \vec{R}_{j}\left(t_{0}\right)}\right\rangle
$$

and Fig. 8 depicts the results obtained for several $q$ values. It shows the usual monotonic decay with time; but comparison with the liquid simple metals (i.e. alkali, alkali-earths, $\mathrm{Al})^{20.26 .30}$ near their triple point shows that at similar $q / q_{p}$ values, the $F_{s}(q, t)$ for l-Si decays much faster. As explained later, this fast decay is related to the significantly greater self-diffusion coefficient in l-Si.

Closely related to $F_{s}(q, t)$ is the velocity autocorrelation function (VACF) of a tagged ion in the fluid, $Z(t)$, which can be obtained as the $q \rightarrow 0$ limit of the first-order memory function of the $F_{s}(q, t)$. However, in the present 


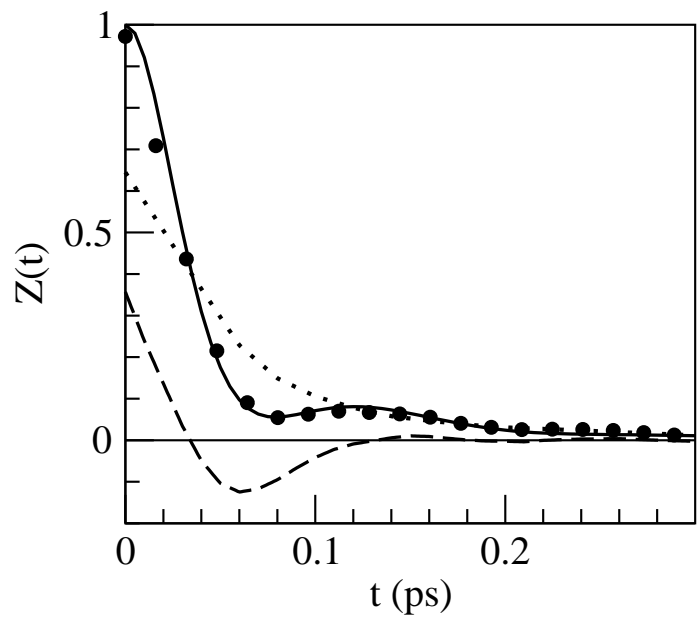

FIG. 9: Normalized OF-AIMD $Z(t)$ for l-Si at $1740 \mathrm{~K}$ (full line). The dashed and dotted lines are the longitudinal, $Z_{l}(t)$, and transverse, $Z_{t}(t)$, components respectively, as defined in Eq. (15), and the full circles represent their sum.

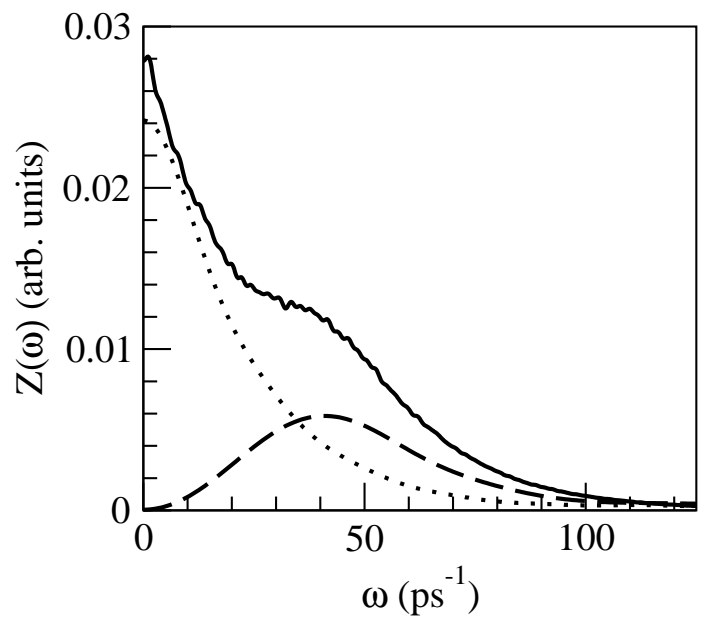

FIG. 10: Same as in the previous figure but for the corresponding power spectrum $Z(\omega)$.

simulations it is more easily obtained from its definition

$$
Z(t)=\left\langle\vec{v}_{1}(t) \vec{v}_{1}(0)\right\rangle /\left\langle v_{1}^{2}\right\rangle
$$

Figures 910 show, respectively, the $Z(t)$ and its power spectrum $Z(\omega)$ obtained by taking the Fourier transform (FT) of the $Z(t)$. Besides the expected diffusive modes at low frequencies, the $Z(\omega)$ shows a shoulder at $\omega \approx 35$ $\mathrm{ps}^{-1}$, which is close to the optical vibrational frequency of covalent $\mathrm{Si}$, and it has been related to the existence of vibrational modes echoing the remnant covalent bonds in the liquid ${ }^{7}$.

The $Z(t)$ shown in Fig. 9 lacks the usual backscattering behaviour observed in the liquid simple metals near melting which is usually ascribed to the so-called "cage effect" due to the shell of nearest neighbors. The relatively open structure of 1 -Si with $\approx 6$ nearest neighbors gives rise to a negligible "cage effect" and $Z(t)$ goes monotonically to zero. Indeed, its shape is remarkably similar to that from the KS-AIMD calculations of Stich et $a^{77.8}$. The self-diffusion coefficient, $D$, is readily obtained from either the time integral of $Z(t)$ or from the slope of the mean square displacement $\delta R^{2}(t) \equiv\left\langle\left|\vec{R}_{1}(t)-\vec{R}_{1}(0)\right|^{2}\right\rangle$ of a tagged ion in the fluid, as follows

$$
D=\frac{1}{\beta m} \int_{0}^{\infty} Z(t) d t ; \quad D=\lim _{t \rightarrow \infty} \delta R^{2}(t) / 6 t
$$

Both routes lead to practically the same value for $D_{\text {OF-AIMD }}=2.28 \AA^{2} /$ ps. We know of no experimental results for the diffusion coefficients of l-Si at any thermodynamic state, but note that the KS-AIMD calculations of Stich et $a{ }^{\pi 7.8}$ gave $D_{\mathrm{KS}-\mathrm{AIMD}}=2.02 \AA^{2} / \mathrm{ps}$. which slightly increased to $2.4 \AA^{2} / \mathrm{ps}$. when the number of particles was increased to 350 particles. They found ${ }^{8}$ that a spin dependent treatment of electron exchange and correlation within the GGA led to a further increase to $D_{\mathrm{KS}-\mathrm{AIMD}}=3.1 \AA^{2} /$ ps., which was explained by a weakening of the interatomic bonds in comparison with the LDA treatment. The KS-AIMD study of Chelikowsky et $a^{19}$ yielded $D_{\mathrm{KS}-\mathrm{AIMD}}=1.90$ $\AA^{2} /$ ps. The present OF-AIMD result remains within the set of values predicted by the KS-AIMD method. Other calculations based on tight binding or interatomic pair potentials 2.5 .6 tend to produce significatively smaller values for the self-diffusion coefficient along with a VACF which takes negative values. We note that $a b$-initio estimates of the self-diffusion coefficient of l-Si are nearly one order of magnitude greater than corresponding ones for the liquid simple metals (alkalis, alkali-earths, $\mathrm{Al}$ ) near melting 20.45 .46 . This larger $D$ explains why $F_{s}(q, t)$ decays, for any $q$-value, much faster than in the simple liquid metals. The accurate gaussian approximation 26.41 gives $F_{s}(q, t)=\exp \left[-q^{2} \delta R^{2}(t) / 6\right]$, and a greater $D \mathrm{im}$ plies a larger $\delta R^{2}(t)$ and a faster decay for the $F_{s}(q, t)$.

With knowledge of the self-diffusion coefficient $D$ and regarding the motion of an atom as the Brownian motion of a macroscopic particle of diameter $d$, the StokesEinstein (SE) relation, $\eta D=k_{B} T / 2 \pi d$ can be used to obtain an estimate for the viscosity $\eta$. Identifying $d$ with the position of the main peak of $g(r)$ as is commonly done gives $d=2.40 \quad \AA$ and taking $D_{\mathrm{OF}-\mathrm{AIMD}}=2.28 \AA^{2} / \mathrm{ps}$ leads to $\eta=0.69 \mathrm{GPa} \cdot \mathrm{ps}$ which is consistent with the previous OF-AIMD estimate obtained from the transverse current correlation function.

To achieve a deeper physical insight into the features of $Z(t)$, we resort to the mode-coupling (MC) approximation of Gaskell and Miller $\underline{47}$ which provides information about the relative importance of the coupling of the single-particle motion to the collective longitudinal and transverse currents. It has already been used used to interpret MD data obtained for the VACF in Lennard- 
Jones fluids, Yukawa fluids, alkali metals and even some molecular liquids ${ }^{47.48 .49}$. Within this approach

$$
\begin{aligned}
Z(t) & \approx \frac{1}{24 \pi^{3}} \int d \mathbf{q} f(q)\left[J_{l}(q, t)+2 J_{t}(q, t)\right] F_{s}(q, t) \\
& \equiv Z_{l}(t)+Z_{t}(t)
\end{aligned}
$$

where $J_{l}(q, t)$ and $J_{t}(q, t)$ are the normalized longitudinal and transverse current correlation functions and

$$
f(q)=\frac{3}{\rho_{i}} \frac{j_{1}(a q)}{a q}
$$

where $j_{1}(x)$ is the spherical Bessel function of order one and $a$ is an ionic radius given by $(4 / 3) \pi a^{3}=1 / \rho_{i}$. Using the OF-AIMD results for $J_{l}(q, t), J_{t}(q, t)$ and $F_{s}(q, t)$ in Eq. (15) allows the contributions of the longitudinal and transverse currents to be separated as $Z_{l}(t)$ and $Z_{t}(t)$ respectively. Firstly we note that, as shown in Fig. 9 the $Z(t)$ obtained by applying Eq. (15) is in excellent agreement with the $Z(t)$ given by the OF-AIMD simulation; therefore it may be reliably used to get deeper insight into the behavior of the $Z(t)$ by analyzing the separate contributions $Z_{l}(t)$ and $Z_{t}(t)$ to the integral (15).

These contributions to $Z(t)$ are plotted in Fig. [9] which shows that $Z_{l}(t)$ has some oscillatory behaviour, but $Z_{t}(t)$ remains positive for all times as a consequence of the mostly positive values taken by the $J_{t}(q, t)$ 's (see Fig. [7. The positive bump of $Z(t)$ arises from the fast decay of $Z_{l}(t)$ which after a negative minimum at a rather short time $(\approx 0.06 \mathrm{ps})$ quickly goes to zero. This behaviour, along with the comparatively slower decay of $Z_{t}(t)$ leads to the bump at $\approx 0.12 \mathrm{ps}$. At longer times, the dynamics of $Z(t)$ is completely determined by $Z_{t}(t)$. This shape has strong similarities with the MD simulation results for liquid water at room conditions ${ }^{49}$, although the $Z(t)$ of l-Si always remains positive whereas in liquid water it takes negative values after the bump. However, in both systems the $Z(t)$ is strongly influenced by $Z_{t}(t)$. In contrast, the simple liquid metals show a very different pattern 47 with both $Z_{l}(t)$ and $Z_{t}(t)$ oscillating about zero. The $Z(t)$ also shows oscillations which, beyond the first minimum, are primarily determined by the $Z_{l}(t)$ which also dominates the large $t$ behaviour of $Z(t)$.

The longitudinal and transverse components of the power spectrum are shown in Fig. 10. The small- $\omega$ behaviour is completely dominated by the $Z_{t}(\omega)$, whereas the shoulder in the $Z(\omega)$ is induced by the maximum in $Z_{l}(\omega)$ at $\omega \approx 40 \mathrm{ps}^{-1}$. Note that the absence of a peak in $Z_{t}(\omega)$ can be traced back to the lack of inelastic peaks in $J_{t}(q, \omega)$ (see Fig. 7), which is in turn an expression of the failure of l-Si to sustain shear waves.

Again, there are substantial differences between the dynamical results for l-Si and those for liquid simple metals for which both $Z_{t}(\omega)$ and $Z_{l}(\omega)$ have clear maxima at $\omega_{t}^{m}$ and $\omega_{l}^{m}$ with $\omega_{t}^{m} \leq \omega_{E} \leq \omega_{l}^{m}$, where $\omega_{E}$ is the socalled "Einstein frequency" of the metal. ${ }^{26}$ Consequently,

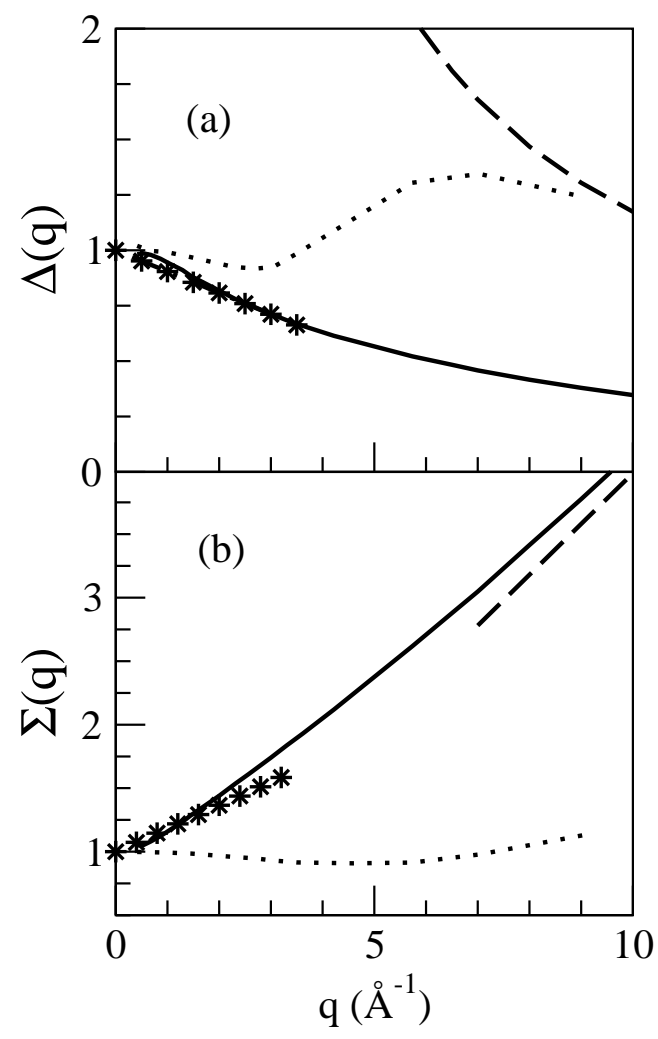

FIG. 11: (a) Normalized HWHM of $S_{s}(q, \omega)$, relative to its value at the hydrodynamic limit, for $\mathrm{l}-\mathrm{Si}$ at $\mathrm{T}=1740 \mathrm{~K}$ (continuous line) and liquid $\mathrm{Al}$ near melting (dotted line). The asteriks are the predictions of the mode-coupling theory and the dashed line gives the free-particle limit. (b) Same as above, but for the normalized peak value $S_{s}(q, \omega=0)$, relative to its value at the hydrodynamic limit.

the $Z(\omega)$ for liquid metals usually has a maximum at $\approx$ $\omega_{t}^{m}$ and a shoulder at $\approx \omega_{l}^{m}$ which, incidentally, is also the pattern exhibited by the MD results for liquid water ${ }^{49}$. In their study of the $Z(t)$ for simple liquids $\frac{47}{4}$, Gaskell and Miller, suggested that the appearance of a peak in $Z(\omega)$ at a frequency well below $\omega_{E}$ was indirect evidence that the liquid system can sustain the propagation of shear waves. The present results which show that l-Si lacks both the frequency peak and shear waves, are consistent with their suggestion. Finally, we note that the diffusion coefficient, which is proportional to $Z(\omega=0)$, is completely determined by the transverse component which contributes $\approx 99 \%$ of the total.

The time $\mathrm{FT}$ of $F_{s}(q, t)$ gives its frequency spectrum, $S_{s}(q, \omega)$, known as the self-dynamic structure factor, which has experimental importance due to its connection with the incoherent part of the INS cross-section. The $S_{s}(q, \omega)$ exhibits, for all $q$-values, a monotonic decay with frequency from a peak value at $\omega=0$. Usually, $S_{s}(q, \omega)$ is characterized by the peak value, $S_{s}(q, \omega=0)$, and the HWHM, $\omega_{1 / 2}(q)$, which are frequently reported normalized with respect to the values of the hydrodynamic $(q$ 
$\rightarrow 0$ ) limit, by introducing the dimensionless quantities $\Sigma(q)=\pi q^{2} D S_{s}(q, \omega=0)$ and $\Delta(q)=\omega_{1 / 2}(q) / q^{2} D$. The OF-AIMD results for $\Delta(q)$ and $\Sigma(q)$ are shown in Fig. 11 along with OF-AIMD results for liquid Al for comparison. Both magnitudes exhibit a behaviour completely different from that of liquid $\mathrm{Al}$ which, in turn, stands as typical of the simple liquids near melting. Indeed, it has been found 20.26 .27 that liquid simple metals near their triple point have an oscillating $\Delta(q)$, stemming from the "cage effect", whereas for a dense gas it decreases monotonically from unity at $q=0$ to a $1 / q$ behaviour at large $q$. The results for l-Si for both $\Delta(q)$ and $\Sigma(q)$ are more similar to those for a dense gas, which must be a consequence of the open structure. An indirect check on the reliability of these results may be provided by the MC theory ${ }^{50.51}$ which, has already shown its capability to account for the experimental $\Delta(q)$ and $\Sigma(q)$ in liquid simple metals 52.53 at $q \leq q_{p}$. According to the MC theory

$$
\begin{gathered}
\Delta(q)=1+H(\delta) q / q^{*} \\
\Sigma(q)=1+G\left(\delta^{-1}\right) q / q^{*}
\end{gathered}
$$

for small $q$-values, where $q^{*}=16 \pi m \rho_{i} \beta D^{2}, \delta=D /(D+$ $\left.\eta / m \rho_{i}\right)$ and the functions $H(\delta)$ and $G\left(\delta^{-1}\right)$ are given in Ref. 52. In both equations the first term is the hydrodynamic result and the second one accounts for the coupling of mass diffusion with the collective modes. The OF-AIMD results for $D$ and $\eta$ have been used to determine $\Delta(q)$ and $\Sigma(q)$ given by Eq. (17) and the results are also shown in Fig. [1] For $q \leq q_{p}$ the OF-AIMD and $\mathrm{MC}$ theory results are in good agreement, similar to that found for simple metals. This agreement points to the ability of the MC theory to describe the single particle dynamics, and presumably the collective dynamics too, in l-Si.

\section{CONCLUSIONS.}

Several static and dynamic properties of l-Si at a thermodynamic state close to the triple point have been evaluated. The simulations have been performed using the orbital free ab-initio molecular dynamics method combined with a first-principles local pseudopotential constructed within the same framework.

The results obtained for the static structure are comparable to those obtained by earlier KS-AIMD calculations ${ }^{7.8 .9}$, and are in reasonable agreement with the available experimental data. We also stress the good description provided by the OF-AIMD method for the orientational correlations, as described by the bond-angle distribution function, $g_{3}\left(\theta, r_{c}\right)$. The obtained structural magnitudes are in vein with other previous ab-initio studies which suggested for l-Si a local structure consisting in a mixture of both tetrahedrally bonded atoms and a higher coordinated structure (probably a distorted metallic white-tin structure). This latter assertion is based on the idea ${ }^{16}$ that for non-close packed systems, the structure of the molten state resembles that of the high pressure solid state. If we now recall that in crystalline Si the semiconducting diamond structure contracts with pressure and transforms at $12 \mathrm{GPa}$ to the metallic white-tin structure, this latter one looks as the most likely to be appear in l-Si.

Although the ab-initio methods based on the KohnSham approach provide a deeper insight into those physical magnitudes related to bonding and electronic properties 7.8 , their heavy computational demands have precluded their application to the study of the dynamic properties of liquids. Indeed, the calculation of the dynamical properties in l-Si has been the main aim of the present calculations which, incidentally were spurred by recent IXS data ${ }^{14,15}$. Moreover, we stress that this is the first ab-initio study on the dynamical properties of 1-Si.

The intermediate scattering functions, $F(q, t)$, have at low- $q$ values, a strong diffusive component which is comparable to what has been found in liquid Ge, but is at variance with the liquid simple metals where the diffusive component is much weaker. The dynamic structure factors, $S(q, \omega)$, show collective density excitations over a similar range of wavelenths as those in liquid simple metals. Moreover, the dispersion relation of the excitations closely follows the existing experimental data14.15.

The transverse current correlation functions, $J_{t}(q, t)$, show extremely weak oscillations around zero and take positive values for most of the time. This shape leads to spectra, $J_{t}(q, \omega)$, with no inelastic peaks which in turn reflect the absence of shear waves. This conclusion is reinforced by the analysis of the spectra of the VACF and appears as an effect of the low coordination number with its attendant negligible "cage effect". This is also reflected in the single particle dynamics, as embodied in the VACF and the self-intermediate scattering functions, leading to a behaviour different from that typical of the liquid simple metals near melting. Nevertheless, the MC theory appears capable of accounting for the single-particle dynamics in l-Si. Calculated self-diffusion and shear viscosity transport coefficients were in fair agreement with experiment and/or other ab-initio calculations.

Up to now, the OF-AIMD method has shown its ability to provide an accurate description of the bulk static and dynamic properties in simple liquid systems ${ }^{20.22}$. However, the present results for the static and dynamic properties of 1-Si illustrate the potential of the OF-AIMD method for treating liquid systems with remnants of covalent bonding and therefore open new venues concerning its range of applicability.

\section{Acknowledgements}

This work has been supported by the DGICYT of Spain (MAT2002-04393-C0201) and the NSERC of Canada. DJG acknowledges additional financial support from the Physics Dept. at Queen's University. 
1 P. Hohenberg and W. Kohn, Phys. Rev. 136, 864 (1964); W. Kohn and L.J. Sham, Phys. Rev. 140, A1133 (1965)

2 F.H Stillinger and T.A. Weber, Phys. Rev. B 31, 5262 (1985); J. Q. Broughton and X. P. Li, Phys. Rev. B 35 , 9120 (1987)

3 J. Tersoff, Phys. Rev. B 38, 9902 (1988)

${ }^{4}$ W. Jank and J. Hafner, Phys. Rev. B 41, 1497 (1990)

5 C. Wang, C Chan and K. Ho, Phys. Rev. Letters 66, 189 (1991); Phys. Rev. B 45, 12227 (1991)

6 R. Virkkunen, K. Laasonen and R M Nieminen, J. Phys.: Cond. Matter 3, 7455 (1991)

7 I. Stich, R. Car and M. Parrinello, Phys. Rev. Letters 63, 2240 (1989); Phys. Rev. B 44, 4262 (1991); I. Stich, Phys. Rev. A 44, 1401 (1991)

8 I. Stich, M. Parrinello and J.M. Hollender, Phys. Rev. Letters 76, 2077 (1996);

${ }^{9}$ V.Godlevsky, J. Chelikowsky and N Troullier, Phys. Rev. B 52, 13281 (1995)

10 J. P. Gabathuler and S. Steeb, Z. Naturforsch 34a, 1314 (1979)

11 Y. Waseda and K. Suzuki, Z.Phys. B 20, 339 (1975); Y. Waseda, The Structure of Non-Crystalline Materials, (New York: McGraw-Hill, 1980)

12 Y. Waseda, K. Shinoda, K. Sugiyama, S. Takeda, K. Terashima and J.M. Toguri, Jpn. J. Appl. Phys. 34, 4124 (1995)

13 S. Takeda, Jpn. J. Appl. Phys. 34, 4889 (1995)

14 S. Hosokawa, J. Greif, F. Demmel and W.-C. Pilgrim, Nucl. Instr. and Meth. in Phys. Res. 199B, 161 (2003)

15 S. Hosokawa, W.-C. Pilgrim, Y. Kawakita, K. Ohshima, S. Takeda, D. Ishikawa, S. Tsutsui, Y Tanaka and A.Q.R. Baron, J. Phys.: Cond. Matter, 15 L623 (2003)

16 V.Petkov and G. Yunchov, J. Phys.: Cond. Matter, 610885 (1994); V.Petkov, S. Takeda, Y. Waseda and K. Sugiyama, J. of Non-Cryst. Solids, 16897 (1994); V.Petkov, J. Phys.: Cond. Matter, 75745 (1995)

17 N. Funamori and K. Tsuji, Phys. Rev. Letters 88, 255508 (2002)

18 G.B. Bachelet, D.R. Hamann and M. Schluter, Phys. Rev. B, 264199 (1982)

19 N. Troullier and J. L. Martins, Phys. Rev. A 43, 1993 (1991)

20 D. J. González, L. E. González, J. M. López and M. J. Stott, Phys. Rev. B 65184201 (2002)

${ }^{21}$ F. Perrot, J. Phys: Condens. Matter 6, 431 (1994); E. Smargiassi and P. A. Madden, Phys. Rev. B 49, 5220 (1994); M. Foley and P. A. Madden, Phys. Rev. B 53, 10589 (1996)

22 J. Blanco, D. J. González, L. E. González, J. M. López and M. J. Stott, Phys. Rev. E 67041024 (2003); D. J. González, L. E. González, J. M. López and M. J. Stott, Phys. Rev. E 69031205 (2004)

23 V.V. Baidov and M.B. Gitis, Sov. Phys. Semicond. 4825 (1970)[ Fiz. Tekh. Poluprovodn. 4967 (1970)]

24 N.E. Cusak, The Physics of Structurally Disordered Matter, (Bristol: Adam-Hilger, 1987)

25 R.L McGreevy, A. Baranyai and I. Ruff, Phys. Chem. Liq., 1647 (1986)

${ }^{26}$ U. Balucani and M. Zoppi, Dynamics of the Liquid State, Clarendon, Oxford, 1994 ; J.P. Hansen and I.R. McDonald, Theory of Simple Liquids, Academic Press, London, 1986
27 A. Torcini, U. Balucani, P.H.K. de Jong and P. Verkerk, Phys. Rev. E 51, 3126 (1995)

${ }^{28}$ F. Shimojo, K. Hoshino and M. Watabe, J. Phys. Soc. Jpn 63141 (1994)

29 S. Kambayashi and G. Kahl, Phys. Rev. A 46, 3255 (1992); G. Kahl and S. Kambayashi, J. Phys.: Cond. Matter, 6 10897 (1994)

30 J. Casas, D. J. González and L. E. González; Phys. Rev. B 60, 10094 (1999); J. Casas, D. J. González, L. E. González, M. M. G. Alemany and L. J. Gallego, Phys. Rev. B 62, $12095(2000)$

31 J.D. Chai, D. Stroud, J. Hafner and G. Kresse, Phys. Rev. B 67, 104205 (2003)

${ }^{32}$ K. Yamamoto, T. Abe and S. Takasu, Jpn. J. Appl. Phys. 30, 2423 (1991)

33 J. G. Cook and G.H. Fritsch, Handbook of Thermodynamic and Transport Properties of Alkali Metals edited by R. W. Ohse (Oxford: Blackwell, 1985), pag 735

34 N. Yoshimoto, M. Ikeda, M. Yoshizawa and S. Kimura, Physica B 219-220, 623 (1996)

35 T. Scopigno, U. Balucani, G. Ruocco and F. Sette, Phys. Rev. E 63, 011210 (2000)

${ }^{36}$ H. Sinn and E. Burkel, J. Phys.: Condens. Matter 8, 9369 (1996); H. Sinn, F. Sette, U. Bergmann, Ch. Halcoussis, M. Krisch, R. Verbeni and E. Burkel, Phys. Rev. Lett. 78, 1715 (1997)

37 C. Pilgrim, S. Hosokawa, H. Saggau, H. Sinn and E. Burkel, J. Non-Cryst. Solids 250-252, 96 (1999)

38 Y. Kawakita, S. Hosokawa, T. Enosaki, K. Ohshima, S. Takeda, C. Pilgrim, S. Tsutsui, Y. Tanaka and A.Q.R. Baron, J. Phys. Soc. Jpn 72, 1603 (2003)

39 S. Hosokawa, H. Sinn, F. Hensel, A. Alatas, E.E. Alp and C. Pilgrim, J. Non-Cryst. Solids 312-314, 163 (2002)

40 I. Ebbsjo, T. Kinell and J. Waller, J. Phys. C 13, 1865 (1980)

41 J. P. Boon and S. Yip, Molecular Hydrodynamics, Dover, New York, 1991

42 B.J. Palmer, Phys. Rev. E 49, 359 (1994)

${ }^{43}$ U. Balucani, J. P. Brodholt, P. Jedlovszky and R. Vallauri, Phys. Rev. E 62, 2971 (2000)

44 H. Sasaki, E. Tokizaki, X.M. Huang, K. Terashima and S. Kimura, Jpn. J. Appl. Phys. 34, 3432 (1995); S. Kimura and K. Terashima, J. of Crystal Growth 180, 323 (1997)

45 T. Iida and R.I.L. Guthrie, Physical Properties of Liquid Metals, Clarendon Press, Oxford, 1988

46 M. M. G. Alemany, J. Casas, C. Rey, L. E. González and L. J. Gallego, Phys. Rev. E 56, 6818 (1997)

47 T. Gaskell and S. Miller, J. Phys. C: Solid St. Phys. 11, 3749 (1978); ibid 11, 4839 (1978); Physics Letters A 66, 307 (1978)

48 J.L. Barrat, J.P Hansen and H. Totsuji, J. Phys. C: Solid State Phys., 214511 (1988)

49 U. Balucani, J. P. Brodholt and R. Vallauri, J. Phys.: Cond. Matter, 86139 (1996)

50 L. Sjögren, J. Phys. C, 13705 (1980); Phys. Rev. A 22, 2866 (1980); Phys. Rev. A 22, 2883 (1980)

51 G Wahnström and L. Sjögren, J. Phys. C, 15401 (1982)

52 W. Moontfrooy, I. de Schepper, J. Bosse, W. Glaser and Ch. Morkel, Phys. Rev. A 33, 1405 (1986)

53 C. Cabrillo, F.J. Bermejo, M. Alvarez, P. Verkerk, A. Maira-Vidal, S.M. Bennington and D. Martin, Phys. Rev. 
Letters 89, 075508 (2002); J. Phys.: Cond. Matter, 16 S309(2004) 